\begin{tabular}{|c|c|c|c|c|c|c|}
\hline \multirow{4}{*}{ Impact Factor: } & ISRA (India) & $=3.117$ & SIS (USA) & $=0.912$ & ICV (Poland) & $=6.630$ \\
\hline & ISI (Dubai, UAE & $=0.829$ & РИНЦ (Russia) & $=0.156$ & PIF (India) & $=1.940$ \\
\hline & GIF (Australia) & $=0.564$ & ESJI (KZ) & $=8.716$ & IBI (India) & $=4.260$ \\
\hline & JIF & $=1.500$ & SJIF (Morocco) & $=5.667$ & OAJI (USA) & $=0.350$ \\
\hline
\end{tabular}

\section{SOI: $\underline{1.1 / \mathrm{TAS}}$ DOI: $\underline{10.15863 / \mathrm{TAS}}$ \\ International Scientific Journal Theoretical \& Applied Science}

\author{
p-ISSN: 2308-4944 (print) e-ISSN: 2409-0085 (online) \\ Year: 2019 Issue: $07 \quad$ Volume: 75
}

Published: $12.07 .2019 \quad$ http://T-Science.org

\section{QR - Issue}

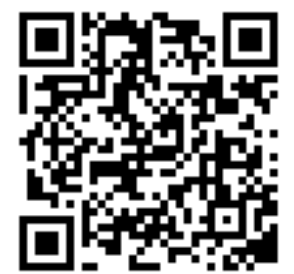

QR - Article
Vadim Andreevich Kozhevnikov
Peter the Great St.Petersburg Polytechnic University
Senior Lecturer
vadim.kozhevnikov@ gmail.com

Alina Alekseevna Shmagina

Peter the Great St.Petersburg Polytechnic University student

\title{
THE SYSTEM OF AUTOMATIC CHECK OF WORK TO NORMALIZE RELATIONS
}

Abstract: We have developed a system designed for students to perform work on the normalization of relations and the subsequent automatic verification by the teacher of this work. The software allows to speed up the testing work and to exclude the subjectivity of the assessment. The work assessment algorithm developed and implemented in the program allows you to point out errors in the student's work and display a message if the work is not credited. This program allows you to perform the normalization of relations and can be used not only for training purposes, but in designing databases.

Key words: automatic check of normalization of relations, relational database, normalization of databases, normal form, functional dependence, decomposition.

Language: Russian

Citation: Kozhevnikov, V. A., \& Shmagina, A. A. (2019). The system of automatic check of work to normalize relations. ISJ Theoretical \& Applied Science, 07 (75), 101-108.

Soi: http://s-o-i.org/1.1/TAS-07-75-18 Doi: crossef https://dx.doi.org/10.15863/TAS.2019.07.75.18

Classifiers: Computer science, computer engineering and automation.

\section{СИСТЕМА АВТОМАТИЧЕСКОЙ ПРОВЕРКИ РАБОТ ПО НОРМАЛИЗАЦИИ ОТНОШЕНИЙ}

Аннотация: Описана система, предназначенная для выполнения студентами работы по нормализации отношений и последующей автоматической проверки преподавателем этой работы. Программа дает возможность ускорить проверку работ и исключить субъективность оценки. Разработанный и реализованный в программе алгоритм оченивания работы позволяет указать на ошибки в работе студента и вывести сообщение в случае, если работа не зачтена. Данная программа позволяет выполнять нормализацию отношений и может быть использована не только в обучающих целях, но и при проектировании баз данньх.

Ключевые слова: автоматическая проверка нормализации отночений, реляционная база данных, нормализация баз данных, нормальная форма, функцииональная зависимость, декомпозиция.

\section{Введение}

Нормализация отношений является очень важной частью процесса проектирования баз данных. В процессе обучения студентов по дисциплинам, связанных с проектированием баз данных, проводятся проверочные работы по нормализации отношений. Работы выполняются в письменном виде и сдаются на проверку преподавателю. Проверка выполненных работ занимает немало времени (с учетом переписываний, их количество может достигать несколько сотен в семестр) и не исключает возможность допущения ошибок при проверке. Поэтому целью создания системы является 


\begin{tabular}{|c|c|c|c|c|c|c|}
\hline \multirow{4}{*}{ Impact Factor: } & ISRA (India) & $=3.117$ & SIS (USA) & $=0.912$ & ICV (Poland) & $=6.630$ \\
\hline & ISI (Dubai, UAI & $=0.829$ & РИНЦ (Russia & $=0.156$ & PIF (India) & $=1.940$ \\
\hline & GIF (Australia) & $=0.564$ & ESJI (KZ) & $=8.716$ & IBI (India) & $=4.260$ \\
\hline & JIF & $=1.500$ & SJIF (Morocce & $=5.667$ & OAJI (USA) & $=0.350$ \\
\hline
\end{tabular}

помощь преподавателю при проверке работ, ускорение процесса проверки, избежание возможных ошибок и субъективности при оценивании. В ходе изучения предметной области прямых аналогов подобной системы найти не удалось, поэтому создание данной системы представляется весьма актуальным. Разработанная система по автоматической проверке работ по нормализации отношений позволит существенно сократить время проверки работ, уменьшить нагрузку на преподавателя, исключить возможность человеческого фактора. Для студентов - ускорит процесс выполнения задания, не снижая степени сложности. На базе созданной системы путем небольшой модификации можно создать программу, которая позволит выполнять полуавтоматическую нормализацию отношений в реальном времени, что при применении в учебных целях позволит студентам лучше понять значимость нормализации в проектировании баз данных, и наглядно продемонстрирует процесс нормализации.

\section{Анализ сушествующих подходов к} автоматической нормализации отношений

Для обеспечения целостности данных при проектировании баз данных используется процедура нормализации. Процесс нормализации выявляет зависимости между атрибутами исходного отношения и позволяет снизить избыточность хранимых данных, устранить аномалии добавления, удаления и модификации [1- 3]. Нормализация - это процесс приведения исходного отношения к нормальным формам. Другими словами, нормализация - это разбиение отношения на две и более таблицы, которые обладают лучшими свойствами при добавлении, изменении и удалении данных. Существует семь нормальных форм, но в рамках выполнения данной работы рассматриваются шесть - 1 НФ, 2 НФ, 3 НФ, НФБК, 4НФ, 5НФ. Мы не будем приводить здесь определения нормальных форм, подразумевая, что читателю они хорошо известны (см., например [1-3]), напомним только важное свойство нормальных форм, заключающееся в том, что по определению - если отношение находится в какой-то нормальной форме, то оно находится и во всех предыдущих нормальных формах. При декомпозиции отношений не должны теряться атрибуты и данные. Данные можно считать не потерянными в том случае, если возможна обратная операция - по декомпозированным отношениям можно восстановить исходное отношение в точности в прежнем виде. Операцией, обратной операции проекции, является операция соединения отношений. Т.к. при восстановлении исходного отношения путем соединения проекций не должны появиться новые атрибуты, то необходимо использовать естественное соединение. Таким образом, без дополнительных ограничений на отношение нельзя говорить о декомпозиции без потерь. Такими дополнительными ограничениями и являются функциональные зависимости. Основной смысл теоремы Хеза заключается в доказательстве того, что при этом не появятся новые кортежи, отсутствовавшие в исходном отношении.

Современные СУБД не предусматривают автоматизацию процесса нормализации. Следовательно, задача по автоматической нормализации отношений остается актуальной. Мы проанализировали существующие алгоритмы [4-7], которые осуществляют автоматическую нормализацию до третьей нормальной формы или до нормальной формы Бойса-Кодда. Среди существующих подходов в области автоматической нормализации отношений следует отметить работу [8]. В ней описывается работа программы, которая автоматизирует процесс нормализации отношения по алгоритму И.А. Зорина. Алгоритм основан на графовом анализе и позволяет формализовать процесс выполнения нормализации реляционной базы данных. Однако рассмотренный алгоритм имеет существенное ограничение функциональности: он обеспечивает перевод проектируемой БД к третьей нормальной форме на основе рассмотрения в предметной области только нетривиальных зависимостей вида $\mathrm{A} \rightarrow \mathrm{C}$ и не устраняют возможные многозначные зависимости, например, между ключевым (А) и неключевым (С) атрибутами в приведенном к ЗНФ отношении (т.е. если семантически С является многозначным атрибутом для некоторого объекта). Приведение БД к нормальной форме Бойса-Кодда (НФБК) с использованием этого алгоритма невозможно. Данный программный продукт позволят автоматически нормализовать отношение. Все рассмотренные нами алгоритмы по автоматической нормализации приводят исходное отношение только к ЗНФ или, в лучшем случае, к НФБК. Как пишут авторы алгоритмов, приведение исходного отношения до ЗНФ вполне достаточно для поддержания целостности базы данных. Однако, задача, которая поставлена в данной работе, несколько отличается от задачи, которую решают эти алгоритмы. Задача нашей системы состоит не только и не столько в нормализации исходного отношения и приведения его к максимально возможной нормальной форме, но и, главное, в оценке, насколько студент может самостоятельно определить потенциальные ключи и функциональные зависимости отношения для каждой нормальной формы. Задача состоит в том, чтобы предоставить возможность студенту 


\begin{tabular}{|c|c|c|c|c|c|c|}
\hline \multirow{4}{*}{ Impact Factor: } & ISRA (India) & $=3.117$ & SIS (USA) & $=0.912$ & ICV (Poland) & $=6.630$ \\
\hline & ISI (Dubai, UAI & $=0.829$ & РИНЦ (Russia & $=0.156$ & PIF (India) & $=1.940$ \\
\hline & GIF (Australia) & $=0.564$ & ESJI (KZ) & $=8.716$ & IBI (India) & $=4.260$ \\
\hline & JIF & $=1.500$ & SJIF (Morocce & $=5.667$ & OAJI (USA) & $=0.350$ \\
\hline
\end{tabular}

самому провести нормализацию. В процессе работы над системой появилась идея создания полуавтоматической нормализации исходной таблицы, которая позволила бы проводить нормализацию от формы к форме поэтапно. Новые экземпляры отношений формируются исходя из указанных функциональных зависимостей. Если согласно указанным Ф3 декомпозиция не выполнилась, то программа сообщает об ошибке выбранных зависимостей. При выполнении работы от студента требуется понимание, как именно происходит переход от одной нормальной формы к другой. Разработанное приложение как раз и отвечает этим требованиям.

\section{Разработка архитектуры системы}

Разрабатываемая система должна обеспечивать взаимодействие трех составляющих - студент, преподаватель и задание. Задание представляет собой исходное отношение (таблицу) и текст задания, описывающий ограничения, накладываемые на исходное отношение. На выходе система должна выдать результат проверки работы студента. Задача внутри системы разбивается на две составляющие. С одной стороны, это взаимодействие студентсистема, где исходными данными является задание, а результатом - выполненная работа студента. С другой стороны, это взаимодействие преподаватель - система, где исходными данными будут выполненная работа студента и знание о правильном решении, а итогом взаимодействия будет проверка работы с выводом соответствующих результатов.

Как уже отмечалось, задача стоит в том, чтобы не только проверить правильность проведенной нормализации, но и оценить выполненную работу. Оценивание выполненного задания происходит по определенным критериям и не все допущенные ошибки являются показателем неверно выполненной работы (оцениваемой на неудовлетворительно). Выполнение нормализации по какому-либо алгоритму позволит автоматически нормализовать отношение, но оно не сможет дать оценку выполненной работе. Тем более что верных решений при нормализации может быть несколько (например, если в исходном или получившемся на каком-то шаге нормализации отношении несколько потенциальных ключей). Поэтому разрабатываемое приложение выполняет две функции. С одной стороны, это пошаговое выполнение нормализации, где приведение к следующей нормальной форме должно осуществляется пользователем, так как проверяется умение нормализовать отношение. С другой стороны, это проверка проведенной нормализации. И для осуществления этой проверки в любом случае необходимо указать исходные данные - зависимости и ограничения. В результате произведенного анализа, лучшим вариантом решения было выбрано сравнение выполненной работы студента с эталонным решением преподавателя, которое записано в файл.

Для дальнейшей разработки системы надо понимать, какие требования предъявляются к выполнению задания, как должно выглядеть выполненное студентом задание. Как уже говорилось, задание - это исходное отношение, то есть таблица. Нормализация - это разбиение исходной таблицы на две и более. На каждом этапе приведения к следующей нормальной форме для каждой таблицы необходимо указать первичный ключ, набор альтернативных ключей и функциональные зависимости. Последующие таблицы формируются исходя из выявленных функциональных зависимостей. Следовательно, в итоге должен получиться некий набор таблиц и для каждой должна сохраняться информация о ключах и зависимостях. Для того, чтобы студент смог выполнить задание, необходима визуализация исходного отношения и визуализация экземпляров отношений, которые будут получены на каждом этапе приведения исходного отношения к следующей нормальной форме. А так же необходим некий интерфейс для ввода студентом информации по каждой таблице, с возможностью последующего сохранения этих параметров. Задание по нормализации отношений для выполнения студентами представляет собой таблицу и текст. Эту таблицу необходимо какимто образом передать в систему для дальнейшей обработки, то есть осуществить с помощью системы приведение исходного отношения к пятой нормальной форме. Как вариант решения этой задачи - это загрузка в систему таблицу из файла. Решением такой задачи является разработка программы, которая будет позволять вводить данные пользователям, хранить таблицы, отображать таблицы и сохранять для каждой таблицы информацию о ней, визуализировать процесс нормализации и иметь возможность сохранения всех накопленных данных.

C точки зрения взаимодействия преподаватель-система исходными данными для системы будет выполненная работа студента. Так как результатом работы системы должна быть проверка выполненного студентом задания, системе каким-то образом необходимо сообщить о том, как правильно это задание должно быть выполнено. То есть, должен быть какой-то правильный, эталонный вариант решения. Для вывода результатов проверки так же требуется визуализация. Необходимо видеть, как студент выполнил задание, и какие данные указывал. При ошибке в каком либо из пунктов работы хотелось 


\begin{tabular}{|c|c|c|c|c|c|c|}
\hline \multirow{4}{*}{ Impact Factor: } & ISRA (India) & $=3.117$ & SIS (USA) & $=0.912$ & ICV (Poland) & $=6.630$ \\
\hline & ISI (Dubai, UAE & $=0.829$ & РИНЦ (Russia & $=0.156$ & PIF (India) & $=1.940$ \\
\hline & GIF (Australia) & $=0.564$ & ESJI (KZ) & $=8.716$ & IBI (India) & $=4.260$ \\
\hline & JIF & $=1.500$ & SJIF (Morocco & $=5.667$ & OAJI (USA) & $=0.350$ \\
\hline
\end{tabular}

бы, чтобы система указала на эту ошибку и вывела общий итог по проверке по системе зачет/незачет. Было бы удобно, если бы преподаватель получал от студента выполненную работу в виде файла. Так же, для удобства и во избежание ошибок, задание студенту может быть передано в виде файла, который загружается в систему.

Исходя из описанного выше, можно сформулировать основные функциональные требования к разрабатываемой системе автоматической проверки работ по нормализации отношений:

- Система должна иметь возможность работы с файлами (открыть, сохранить).

- Должна быть возможность указать информацию о студенте, который выполнил эту работу для последующей идентификации работы.

- Приложение должно хранить таблицы и иметь возможность манипулирования этими таблицами.

- Система должна выполнять визуализацию процесса нормализации.

- Для каждой нормальной формы должна быть возможность отобразить экземпляры отношений, указать для каждого экземпляра потенциальные ключи, выделить среди них первичный ключ, указать функциональные зависимости.

- Для исходного отношения должна быть возможность описать аномалии вставки, удаления, модификации.

- Для каждого экземпляра отношений должна быть возможность указать состояние, в котором находится отношение, и дать объяснение.

- Должна быть возможность просмотра исходного отношения в отдельном окне.

- При осуществлении проверки, система должна указывать, где именно была допущена ошибка.

- Система должна выводить сообщение, зачтена или не зачтена работа.

При выборе формата файлов необходимо учесть особенности использования этих файлов. Когда требуется создать много таблиц, для этого студентам и преподавателям удобнее и привычнее использовать формат файлов Microsoft Excel. Excel позволяет не только легко создать таблицу, но и вставить текст задания. Excel прекрасно подойдет для сохранения выполненного задания, так как это тоже таблицы и небольшой сопутствующий текст.

Для хранения и осуществления манипуляций над таблицами необходима реляционная база данных. Здесь нет необходимости использовать клиент-серверную архитектуру, следовательно, в качестве базы данных для хранения таблиц можно выбрать встраиваемую базу данных, например, SQLite.
Исходя из функциональных требований к системе, можно выделить основные классы, на которые она будет разделена:

- Класс, работающий с файлами типа excel. Класс читает данные из файла и сохраняет данные в файл. Так же в этом классе реализован алгоритм сравнения и оценки работы.

- Класс, обеспечивающий соединение и работу с базой данных SQLite. В этом классе так же создаются объекты, отображающие таблицы из базы данных, которые потом передаются в главное окно приложения и отображаются на форме. Эти объекты хранятся в списках для каждой нормальной формы.

- Основное окно приложения. Здесь студент вводит информацию о себе. Здесь отображаются нормальные формы и экземпляры отношений, сформированные в базе данных для этой нормальной формы.

- Класс для отображения нормальной формы.

- Класс для отображения экземпляра отношения.

\section{Принцип работы системы}

Опишем вначале действия, производимые системой при загрузке задания студентом. Студент нажимает кнопку «Загрузить задание» на главном окне приложения. Через класс, осуществляющий чтение файлов Excel, данные из файла-задания загружаются в программу. В базе данных создается исходное отношение и создается его представление для отображения в окне приложения на форме. Далее создается графический объект, отображающий на главном окне приложения интерфейс для исходного отношения, которое, исходя из условий задания, находится в первой нормальной форме. Объект формы запрашивает список с отображением таблиц, которые предназначены для текущей нормальной формы. Студент нажимает кнопку «Добавить экземпляр отношения» и исходное отношение отображается на форме.

Опишем теперь действия, производимые системой при проверке выполненной работы студента преподавателем. При загрузке файла с выполненной работой студента, сохраняются данные в программе, формируются все нормальные формы, формируются все таблицы и отображаются каждая на своей форме. При загрузке эталонного файла, сохраняются данные файла и осуществляется выполнение алгоритма проверки. При обнаружении ошибок информация о них передается в соответствующие формы.

Важно отметить, что для автоматизации процесса проверки требуется единообразие сбора информации и записи этой информации. При выполнении задания студент заполняет форму, согласно указаниям и тексту приложения. Таким 


\begin{tabular}{|c|c|c|c|c|c|c|}
\hline \multirow{4}{*}{ Impact Factor: } & ISRA (India) & $=3.117$ & SIS (USA) & $=0.912$ & ICV (Poland) & $=6.630$ \\
\hline & ISI (Dubai, UAI & $=0.829$ & РИНЦ (Russia) & $=0.156$ & PIF (India) & $=1.940$ \\
\hline & GIF (Australia) & $=0.564$ & ESJI (KZ) & $=8.716$ & IBI (India) & $=4.260$ \\
\hline & JIF & $=1.500$ & SJIF (Morocco) & $=5.667$ & OAJI (USA) & $=0.350$ \\
\hline
\end{tabular}

образом, информация по каждой таблице будет сохраняться одинаково для всех вариантов.

Сравнение результатов происходит по следующему алгоритму. Вначале происходит загрузка всех данных в программу. Данные по каждой таблице, номер нормальной формы сохраняются в структуры, структуры хранятся в списке. После загрузки данных из файла выполненной работы студента и файла с верным решением, получаются два списка - список со структурами по данным студента и список со структурами по данным преподавателя. Далее программа выполняет циклы. Первый цикл - это перебор нормальных форм. Цикл повторяется шесть раз. Внутри цикла выделяются структуры из списка, которые относятся к текущей итерации цикла, то есть к одной и той же нормальной форме. Создается коллекция для расстановки приоритетов, в которую входят индекс приоритета (key) и номер блока из набора структур преподавателя (value). Создается список совпадений. Этот список содержит в себе структуры с данными о результатах сравнения по одному блоку: номер блока, 0 или 1 для ПК (первичный ключ), 0 или 1 для АК (альтернативный ключ), 0 или 1 для Ф3 (функциональная зависимость). Далее происходит сравнение данных из каждого блока студента с данными из каждого блока преподавателя. Для перебора всех блоков студента служит второй цикл. Третий цикл перебирает блоки преподавателя. Внутри этого цикла происходит проверка, сравнение данных из блока студента и блока преподавателя. Когда все блоки преподавателя будут рассмотрены, структуры с данными по сравнению записываются в список. В итоге получается некая матрица сравнения, пример которой приведен в табл. 1.

Таблица 1. Пример матрицы сравнения

\begin{tabular}{|l|l|l|l|l|}
\hline $\begin{array}{l}\text { Номер блока (таблицы) } \\
\text { преподавателя }\end{array}$ & ПК & АК & Ф3 & Текст \\
\hline 0 & 0 & 0 & 0 & “’” \\
\hline 1 & 1 & 0 & 0 & “’” \\
\hline 2 & 1 & 1 & 1 & “'” \\
\hline
\end{tabular}

Далее необходимо определить, какой из блоков преподавателя наилучшим образом совпал с блоком студента. Так как критерий определения правильного ответа не так очевиден, была выведена таблица приоритета ошибок, которая приведена в табл. 2

Таблица 2. Таблица приоритета ошибок

\begin{tabular}{|l|l|l|l|}
\hline ПК & АК & Ф3 & Приоритет \\
\hline 1 & 1 & 1 & 1 \\
\hline 1 & 0 & 1 & 2 \\
\hline 0 & 0 & 1 & 3 \\
\hline 1 & 1 & 0 & 4 \\
\hline 1 & 0 & 0 & 5 \\
\hline 0 & 0 & 0 & 6 \\
\hline
\end{tabular}

Приоритеты расставлены в соответствии с тем, насколько критична для оценки та или иная ошибка в блоке студента. Согласно этой таблице в качестве верного ответа выбирается тот номер блока, который получил наивысший приоритет. По этому принципу просматриваются все блоки студента. После чего данные по каждому блоку передаются в главное окно приложения, в котором, при возникновении ошибки, выводится сообщение, что работа не зачтена. Напротив тех пунктов, где эта ошибка была допущена, появляются надписи красным цветом о типе ошибки.

\section{Реализация системы}

Система автоматической проверки работ по нормализации отношений реализована на языке программирования $\mathrm{C}++\quad \mathrm{c}$ использованием фреймворка Qt [9] (поскольку в нем есть всё, что необходимо для реализации данного проекта поддержка работы с базами данных, удобное создание графического интерфейса, система 


\begin{tabular}{|c|c|c|c|c|c|c|}
\hline \multirow{4}{*}{ Impact Factor: } & ISRA (India) & $=3.117$ & SIS (USA) & $=0.912$ & ICV (Poland) & $=6.630$ \\
\hline & ISI (Dubai, UAE & $=0.829$ & РИНЦ (Russia & $=0.156$ & PIF (India) & $=1.940$ \\
\hline & GIF (Australia) & $=0.564$ & ESJI (KZ) & $=8.716$ & IBI (India) & $=4.260$ \\
\hline & JIF & $=1.500$ & SJIF (Morocco & $=5.667$ & OAJI (USA) & $=0.350$ \\
\hline
\end{tabular}

сигналов и слотов, упрощающая взаимодействие между компонентами программы). Приложение создавалось в IDE Qt Creator 4.4.1, основанном на версии Qt 5.9.2, компилятор MinGW 5.3.0 32bit.

Исходными данными для выполнения задания студентом с помощью разрабатываемой системы является таблица, которая должна быть помещена в систему и с помощью манипуляций студента преобразована в несколько таблиц, то есть приведена к пятой нормальной форме. После выполнения задания студент сохраняет свою выполненную работу в файл. Преподаватель в качестве исходных данных получает файл с выполненной работой, плюс файл с правильным ответом на эту работу. Задание для студентов выдается индивидуальное, следовательно, преподаватель должен создать некоторое количество разных таблиц. Как уже говорилось, для этого удобнее использовать формат файлов Microsoft Excel. Excel так же позволяет вставить текст задания. Создание и редактирование таблицы при использовании текстового формата файлов, например txt или $\mathrm{xml}$, несколько проблематично и ненаглядно, что будет усложнять задачу преподавателю при формировании задания студентам. С одной стороны, использование формата excel лишает систему кроссплатформенности и привязывает её к работе только с Windows, но с другой стороны, для решения учебных задач, не связанных с другими ОС, вполне достаточно и более привычным для большинства студентов является OC Windows. Выбрав в качестве исходного формата формат excel, целесообразно не переходить к другим форматам файлов и сохранять решенное задание студента так же в формате excel. Использование файлов при работе c программой накладывает определенные ограничения и правила на создание таких файлов. Задание, которое формирует преподаватель должно быть записано в файл формата excel определенным образом. На первом листе в первой ячейке первой строки всегда должно быть название исходного отношения. Начиная со второй строки должна располагаться сама таблица с заголовками. На втором листе размещается текст задания. Записи в файле excel выполненного студентом задания организованы следующим образом: в каждый лист файла записывается определенная информация в строго организованном порядке. Запись данных осуществляется по принципу "ключевое слово""текст". Первый лист содержит информацию о студенте. Номер группы, фамилия, имя, отчество, вариант задания, дата выполнения. Листы со второго по седьмой отведены под запись информации о таблицах для каждой нормальной формы. Помимо информации о таблице, на листе сохраняется текст комментария, который студенты должны давать в качестве пояснения, находится ли данная таблица в текущей нормальной форме. Кроме того, для исходного отношения необходимо указывать примеры аномалий (вставки, обновления и удаления). Этот текст записывается для первой нормальной формы и сохраняется в ехcel только на втором листе. На последнем, восьмом листе файла excel, сохраняется таблица исходного отношения. При вызове из меню программы подменю "Открыть" данные считываются по номерам листов. Сохраненная таблица на последнем листе необходима для построения всех таблиц, на которые оно разбивается в ходе декомпозиции. Аналогичным образом организован файл с верным ответом. Для универсальности нумерация листов и порядок остаются такими же, как и в выполненной работе студента.

Как уже отмечалось, при разработке архитектуры приложения было решено отказаться от клиент-серверной архитектуры в пользу локального приложения с использованием SQLite [10]. SQLite - это компактная встраиваемая реляционная база данных, которая может поставляться вместе с приложением. Использование SQLite делает систему автономной и независимой.

Примеры интерфейса приложения приведены на рис. 1 и рис. 2. 


\begin{tabular}{llllll} 
& ISRA (India) $=\mathbf{3 . 1 1 7}$ & SIS (USA) & $=\mathbf{0 . 9 1 2}$ & ICV (Poland) & $=\mathbf{6 . 6 3 0}$ \\
Impact Factor: & ISI (Dubai, UAE) $=\mathbf{0 . 8 2 9}$ & PUHL (Russia) $=\mathbf{0 . 1 5 6}$ & PIF (India) & $=\mathbf{1 . 9 4 0}$ \\
& GIF (Australia) $=\mathbf{0 . 5 6 4}$ & ESJI (KZ) $=\mathbf{8 . 7 1 6}$ & IBI (India) & $=\mathbf{4 . 2 6 0}$ \\
& JIF & $\mathbf{1 . 5 0 0}$ & SJIF (Morocco) $=\mathbf{5 . 6 6 7}$ & OAJI (USA) & $\mathbf{0 . 3 5 0}$ \\
\hline
\end{tabular}

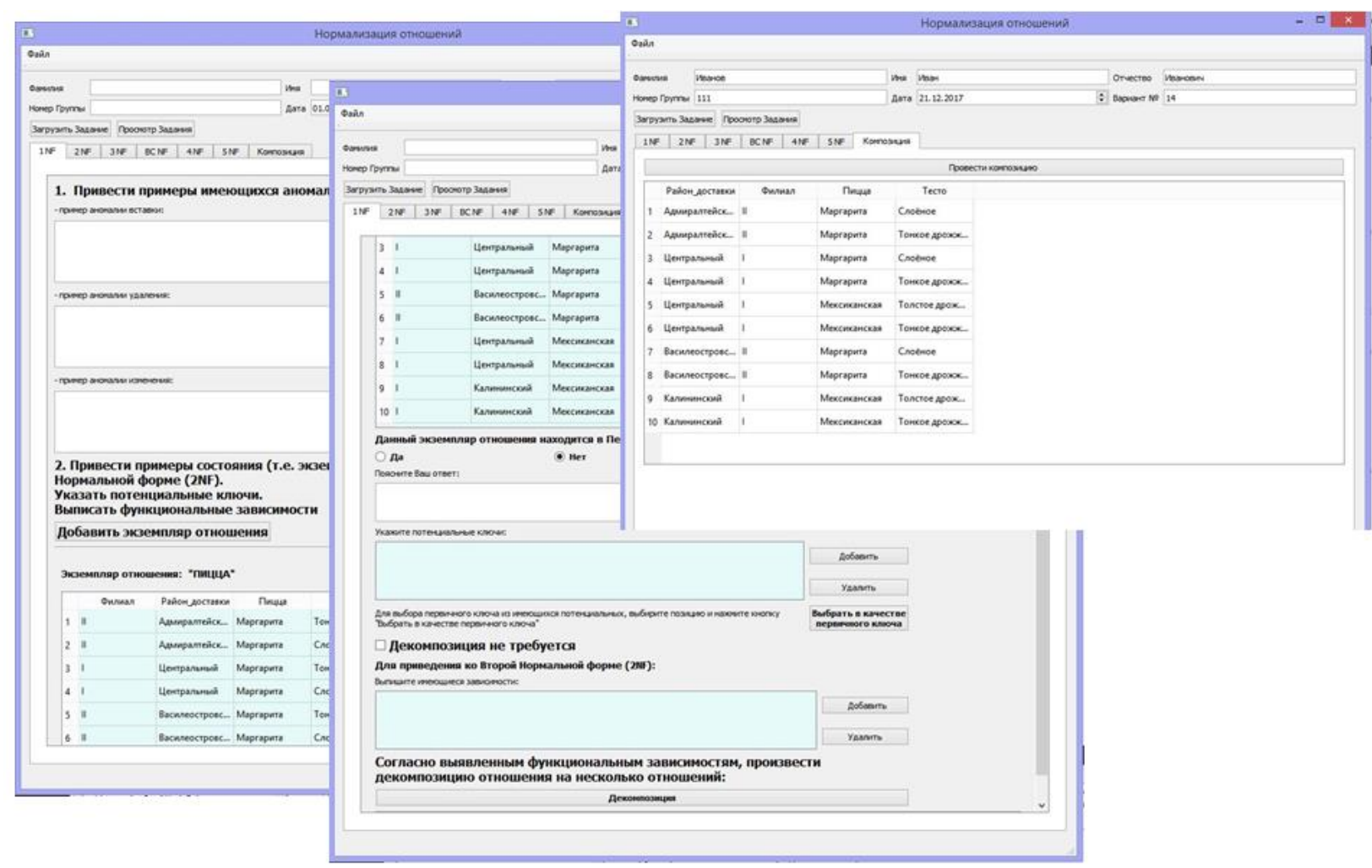

Рисунок 1. Внешний вид приложения

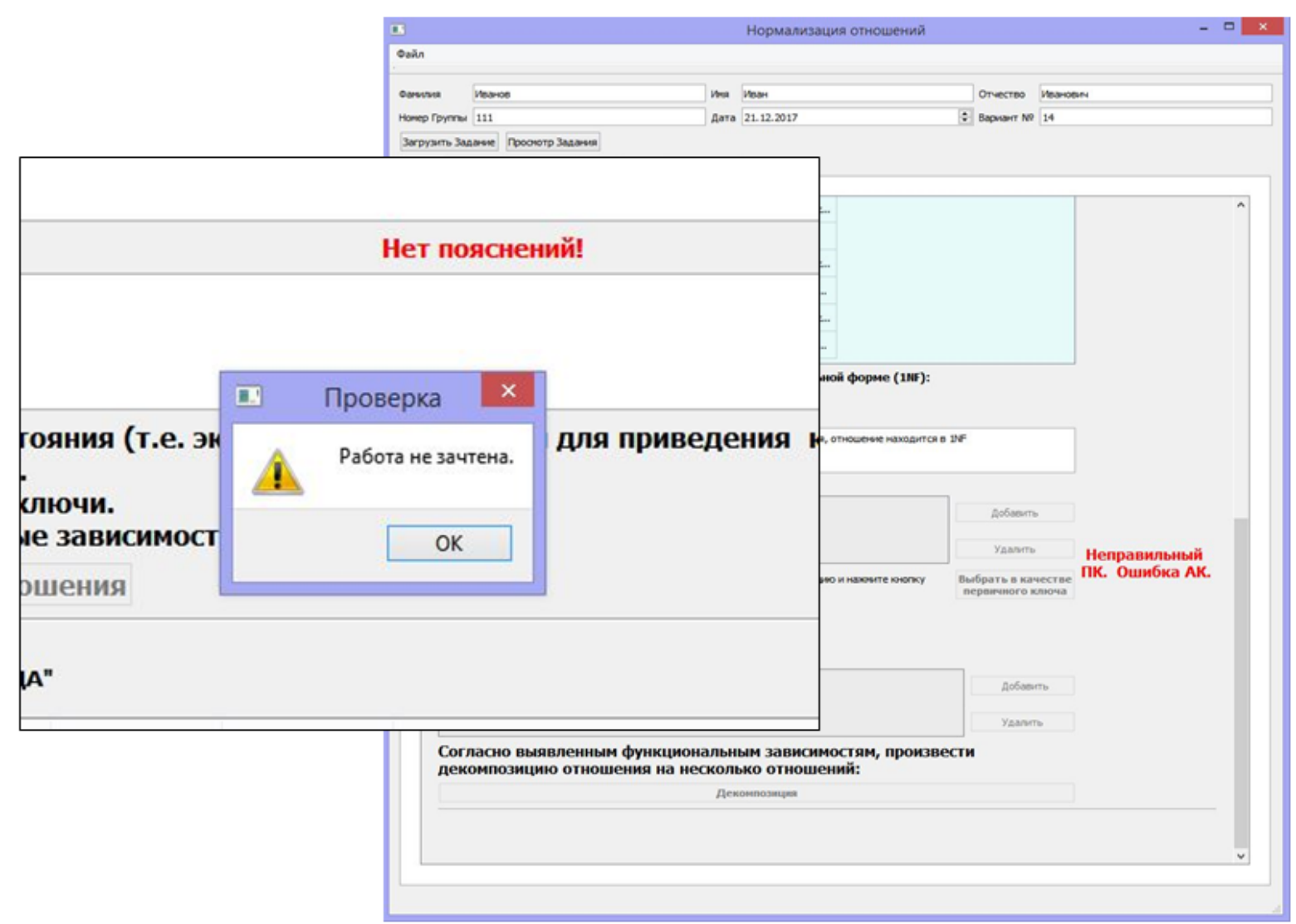

Рисунок 2. Пример проверки выполненной работы 


\begin{tabular}{llllll} 
& ISRA (India) $=\mathbf{3 . 1 1 7}$ & SIS (USA) & $=\mathbf{0 . 9 1 2}$ & ICV (Poland) & $\mathbf{= 6 . 6 3 0}$ \\
Impact Factor: & ISI (Dubai, UAE) $=\mathbf{0 . 8 2 9}$ & PUHL (Russia) $=\mathbf{0 . 1 5 6}$ & PIF (India) & $=\mathbf{1 . 9 4 0}$ \\
& GIF (Australia) $=\mathbf{0 . 5 6 4}$ & ESJI (KZ) & $=\mathbf{8 . 7 1 6}$ & IBI (India) & $=\mathbf{4 . 2 6 0}$ \\
& JIF & $\mathbf{1 . 5 0 0}$ & SJIF (Morocco) $=\mathbf{5 . 6 6 7}$ & OAJI (USA) & $\mathbf{0 . 3 5 0}$ \\
\hline
\end{tabular}

Приложение было протестировано на работах студентов. Результаты автоматической проверки полностью совпадали с результатами проверки преподавателем.

\section{Заключение}

Нами были проанализированы существующие подходы по нормализации отношений. Исследованы возможные варианты решения поставленной задачи и выбран на наш взгляд наиболее удобный и функциональный из них. Разработанная система позволяет с одной стороны выполнять работу по нормализации исходного отношения, с другой стороны автоматически проверить результат выполнения работы студентом. Для проведения автоматической проверки был разработан алгоритм, при выполнении которого не только оценивается работа студента, но и указываются допущенные ошибки.

Помимо проверки работ студентов, разработанный программный продукт может быть использован при проектировании баз данных. Он является независимым приложением и не привязан к конкретной СУБД.

В настоящее время ведется работа по увеличению функциональности системы, наделения её новыми функциями. Например, создание режима, который будет сразу же сообщать пользователю, правильно ли выбран первичный ключ и указаны функциональные зависимости - получится полуавтоматическая система по нормализации отношений. Другой возможный режим программы - это проведение проверки на наличие аномалий в существующем исходном отношении.

\section{References:}

1. Date, C. J. (2003). An Introduction to Database Systems. 8 edition. Pearson, p.1040.

2. Connolly, T. M., \& Begg, C. E. (2009). Database Systems. A Practical Approach to Design, Implementation, and Management. 5th edition. Pearson, p. 1400.

3. Garcia-Molina, H., Ulman, J. D., \& Widom, J. (2011). Database Systems: The Complete Book. 2nd edition. Pearson.

4. Vinogradova, M. V., \& Igushev, E. G. (2011). Konstruktor baz dannykh na osnove sushchnostey i ikh rekvizitov s vozmozhnost'yu normalizatsii. Nauka $i$ obrazovanie, N10, oktyabr'.

5. Zorin, I. A. (2009). Teoretiko-grafovoe privedenie relyatsionnoy bazy dannykh $\mathrm{k}$ tret'ey normal'noy forme E. Kodda. Informatsionnaya matematika, $N 2$ (8)

6. Klimenko, I. V. (2012). Metod formal'noy normalizatsii otnosheniy relyatsionnoy modeli bazy dannykh. Vestnik RGUPS, N2 (46), p.7987.

7. Storozhok, E. A., \& Tarasov, V. N. (2011). Algoritm normalizatsii skhemy relyatsionnoy bazy dannykh. Al'manakh sovremennoy nauki $i$ obrazovaniya. Tambov: Gramota, $N$ 3(46), pp.87-92.

8. Govorova, M. I., Govorov, A. I., \& Maslennikov, R. I. (2015). Programma normalizatsii relyatsionnykh baz dannykh kak osnova predmetno-orientirovannoy intellektual'noy obuchayushchey sistemy. Obrazovatel'nye tekhnologii $i$ obshchestvo, N1 (18), pp.505-518.

9. (n.d.). Qt Documentation [Elektronnyy dokument]. Retrieved July 08, 2019, from http://doc.qt.io .

10. (n.d.). SQLite Documentation [Elektronnyy dokument]. Retrieved July 08, 2019, from http://www.sqlite.org/docs.html 